\title{
Possible Distribution of Magnetic Vortices of Two-Band Mesoscopic Superconductors in Different Regions of Parameter Space
}

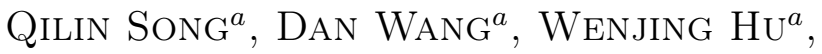 \\ Lin PenG ${ }^{a, *}$, Yun ZHOU ${ }^{b}$, \\ ZHIYONG $\mathrm{LIU}^{c}$ AND JIA LIN ${ }^{a}$ \\ ${ }^{a}$ Department of Physics, Shanghai University of Electric Power, Shanghai 201300, China \\ ${ }^{b}$ Department of Physics, China Jiliang University, Hangzhou 310018, China \\ ${ }^{c}$ Shanghai Key Laboratory of High Temperature Superconductors, Physics Department, \\ Shanghai University, 99 Shangda Road, Shanghai 200444, China
}

Received: 31.12.2020 \& Accepted: 18.03.2021

\begin{abstract}
Using a finite element method to the time-dependent two-band Ginzburg-Landau model we numerically explored the possible distribution of magnetic vortices of two-band mesoscopic superconductors in different regions of the parameters. The parameter space is defined by microscopic quantities such as partial density of states, the Fermi velocity ratio and elements of the coupling matrix. In particular, when the interband coupling and the Fermi velocity ratio are fixed, the critical temperatures of band activity $\left(T_{c 1}\right.$ and $\left.T_{c 2}\right)$ are modulated by changing the partial densities of states, $n_{1}$ and $n_{2}$. Due to simulation results, we obtained possible distribution of magnetic vortices including fractional vortices and composite vortices.
\end{abstract}

topics: fractional vortices, composite vortices, mesoscopic superconductor

\section{Introduction}

Vortex matters in multiband superconductors were intensively studied, especially since their discovery in $\mathrm{MgB}_{2}$ and the new iron-based superconductors [1-7]. Multiband superconductors present a variety of intriguing properties that are not found in single-band superconductors, such as the timereversal symmetry-broken phase [8], short-range repulsion and long-range attraction between vortices [9], oscillations of superconducting phase difference between different bands [10] and fractional vortices [11-15], etc. The appearance of fractional vortices is possible in mesoscopically confined samples, where condensates associated with different bandgaps can have different phase winding numbers due to their coexisting weakly interaction and significantly different coherence length [11].

Multiple condensates simultaneously couple to a common gauge field, and a vortex associated with a $2 \pi$-phase rotation in one condensate only carries a fractional quantum flux [12]. When interband phase differences are locked to each other due to strong couplings, a phase rotation along a closed path is the same for different condensates. Thus, vortices in different bands overlap in space and form the composite vortex with the standard integer quantum flux $[11,12]$. Therefore, the composite vortex can be viewed as a bound state of fractional vortices in different bands with the vortex cores located at the same position. In addition, the composite vortex can be decoupled by the driving current [13] or thermal fluctuations [15] resulting in the fractional vortices.

The fractional states are typically found in limited ranges of applied field, and will have higher energy than the conventional vortex states due to the very weak coupling between the band and the condensates.

In this paper, our interest is to explore possible distribution of fractional vortices of two-band mesoscopic superconductors in different regions of the parameter space by using the time-dependent twoband Ginzburg-Landau (TDTBGL) model, where we have cautiously set the temperature $T$ close to the critical temperature $T_{c}$ in order to ensure the qualitative and quantitative validity of our results $[14,16]$. It is evidenced that standard multiband Ginzburg-Landau model provides quite an accurate quantitative description of the specific 
features of two-band superconductivity [17, 18]. By using the finite-element method (FEM) [19-22], the TDTBGL equations are numerically solved to obtain the vortex properties of mesoscopic two-band superconductors, including fractional vortices and composite vortices.

The paper is organized as follows. In Sect. 2, we show the derived TDTBGL equations and explain the numerical method and procedure used in our calculations. In Sect. 3, we analyze the results obtained for the two-band superconductors. Our results are finally summarized in Sect. 4.

\section{Time-dependent two-band Ginzburg-Landau theory}

We consider a mesoscopic two-band superconducting square of thickness $d$ much smaller than the penetration depth $\lambda$ and the two characteristic lengths of density variations in both condensates, in such a way that the superconducting square is effectively two-dimensional, in the $(x, y)$ plane. The $\psi_{1}$ and $\psi_{2}$ show the wave functions of the first band condensate and the second band condensate, respectively. The $\boldsymbol{H}=(0,0, H)$ is the applied magnetic field, and $\boldsymbol{B}=\nabla \times \boldsymbol{A}$ is the local field. If the superconductor is driven out of equilibrium, the order parameter should relax back to its equilibrium value. The dimensionless TDTBGL equations in the zero-electrostatic potential gauge can be written as follows [14]:

$$
\begin{aligned}
& -\Gamma_{1} \frac{\partial \psi_{1}}{\partial t}=(-\mathrm{i} \nabla-\boldsymbol{A})^{2} \psi_{1} \\
& -\left(\tau_{1}-\left|\psi_{1}\right|^{2}\right) \psi_{1}-\gamma \psi_{2}, \\
& -\Gamma_{2} \frac{\partial \psi_{2}}{\partial t}=\frac{1}{\alpha}(-\mathrm{i} \nabla-\boldsymbol{A})^{2} \psi_{2} \\
& -\left(\tau_{2}-\left|\psi_{2}\right|^{2}\right) \psi_{2}-\frac{k_{2}^{2}}{\alpha^{2} k_{1}^{2}} \gamma \psi_{1}, \\
& \sigma_{n} \frac{\partial \boldsymbol{A}}{\partial t}=\boldsymbol{J}_{s}-\kappa_{1}^{2} \nabla \times \nabla \times \boldsymbol{A}, \\
& \boldsymbol{J}_{s}=\frac{\mathrm{i}}{2}\left(\psi_{1} \nabla \psi_{1}^{*}-\psi_{1}^{*} \nabla \psi_{1}\right)-\left|\psi_{1}\right|^{2} \boldsymbol{A} \\
& +\frac{\alpha k_{1}^{2}}{k_{2}^{2}}\left(\frac{\mathrm{i}}{2}\left(\psi_{2} \nabla \psi_{2}^{*}-\psi_{2}^{*} \nabla \psi_{2}\right)-\left|\psi_{2}\right|^{2} \boldsymbol{A}\right) .
\end{aligned}
$$

Here, one reads $\tau_{j}=-\ln \left(\frac{T}{T_{c j}}\right)=\tau-\frac{S_{j}}{n_{j} \eta}$ and $S_{j}=\lambda_{j j}-n_{j} \eta S$, for $j=1,2$. Furthermore,

$$
\begin{aligned}
S & =\frac{n_{1} \lambda_{11}+n_{2} \lambda_{22}}{2 \operatorname{det}(\Lambda)} \\
& -\frac{\sqrt{\left(n_{1} \lambda_{11}+n_{2} \lambda_{22}\right)^{2}-4 \operatorname{det}(\Lambda)}}{2 \operatorname{det}(\Lambda)}
\end{aligned}
$$

with $\operatorname{det}(\Lambda)=n_{1} n_{2}\left(\lambda_{11} \lambda_{22}-\lambda_{12}^{2}\right)$, and

$$
\tau=-\ln \left(\frac{T}{T_{c}}\right) \simeq 1-\frac{T}{T_{c}} .
$$

The relaxation time of order parameters is denoted by $\Gamma_{1,2}, \sigma_{n}$ is the conductivity of the sample in a two-band case, and $\eta=\operatorname{det}(\Lambda) /\left(n_{1} n_{2}\right)$. The electron-phonon coupling matrix is expressed as

$$
\Lambda=\left(\begin{array}{ll}
n_{1} \lambda_{11} & n_{2} \lambda_{12} \\
n_{1} \lambda_{12} & n_{2} \lambda_{22}
\end{array}\right) .
$$

From the above expressions, one can obtain the intrinsic critical temperatures $T_{c j}=T_{c} \exp \left(-S_{j} /\left(n_{j} \eta\right)\right)$. The strength of the Josephson coupling is determined by $\gamma=\lambda_{12} /\left(n_{1} \eta\right)$. The intrinsic TDTBGL parameters of each band are denoted as

$$
\begin{aligned}
& \kappa_{1}=\frac{3 c W}{h e v_{1}^{2}} \sqrt{\frac{\pi}{2 n_{1} N(0)}}, \\
& \kappa_{2}=\kappa_{1} \alpha \sqrt{\frac{n_{1}}{n_{2}}} .
\end{aligned}
$$

The critical temperature is given by the relation $1.76 T_{c}=2 \hbar \omega_{\mathrm{D}} \mathrm{e}^{-S}$, where $\omega_{D}$ is the Debye frequency [16]. Other relevant quantities are the Fermi velocity of the first band $v_{1}$, the square of the ratio of the Fermi velocities in the two bands $\alpha=\left(v_{1} / v_{2}\right)^{2}$, the elements of the coupling matrix $\lambda_{11}, \lambda_{22}$ and $\lambda_{12}=\lambda_{21}$, the total density of states $N(0)$, the partial density of states of the first band $n_{1}$ (thus $n_{2}=1-n_{1}$ ), and $T_{c}$, which sets the energy scale $W^{2}=\left(4 \pi T_{c}\right)^{2} /(17 \zeta(3))$. Let us take the normalization for the order parameters as $W$, the normalization for the vector potential as $A_{0}=h c /\left(4 e \pi \zeta_{1}\right)$, for the lengths as $\zeta_{1}=\hbar v_{1} /(\sqrt{6} W)$, the normalization for the temperatures as $T_{c}$, and for the time by $t_{0}=4 \pi \sigma k_{1}^{2} \zeta_{1}^{2} / c^{2}$ (where $\sigma$ is the normal-state conductivity). The magnetic field is given in the units of the thermodynamic critical field $H_{c}$. For the magnetic field, the boundary condition reads as follows:

$$
\left.(\nabla \times \boldsymbol{A})\right|_{\text {boundary }}=\boldsymbol{H} \text {. }
$$

For the order parameter, the superconductorinsulator boundary conditions are used

$$
\begin{aligned}
& \left(-\mathrm{i} \nabla \psi_{1}-\boldsymbol{A} \psi_{1}\right) \cdot \boldsymbol{e}_{1}=0, \\
& \left(-\mathrm{i} \nabla \psi_{2}-\boldsymbol{A} \psi_{2}\right) \cdot \boldsymbol{e}_{2}=0,
\end{aligned}
$$

where $\boldsymbol{e}_{1}$ and $\boldsymbol{e}_{2}$ are the outward normal units to the surface. First, we have solved (1)-(4) at zero value of the applied field. Then, the field was adiabatically increased, in small steps $\Delta H=0.005 H_{c}$. The dimensionless magnetization, which is a direct measure of the magnetic field expelled from the system, is defined as $M=\frac{1}{4 \pi}(\langle B\rangle-H)$, where $\langle B\rangle$ denotes the magnetic induction averaged over the mesoscopic superconducting square area $S$.

In order to perform simulations of an evolution of a stable initial state, we need to set the initial condition for $\psi_{1}$ and $\psi_{2}$. In our study, we chose that

$$
\begin{aligned}
& \psi_{1}=\sqrt{n_{1}} \tau_{1}+\mathrm{i} \sqrt{n}_{2} \tau_{1}, \\
& \psi_{2}=\sqrt{n_{1}} \tau_{2}+\mathrm{i} \sqrt{n}_{2} \tau_{2} .
\end{aligned}
$$




\section{Results and discussion}

We consider the mesoscopic superconducting square with sizes of $L_{x} \times L_{y}=30 \zeta_{1} \times 30 \zeta_{1}$. Here, we adopt a different strategy and study the possible configurations of vortex states in different regions of the parameter space defined by the microscopic quantities. For a treatable analysis, we first fix the temperature of the systems at $T=0.9$ to firmly remain in the validity regime of the TDTBGL theory, and choose microscopic parameters such that both $T_{c 1}$ and $T_{c 2}$ are also close to $T_{c}$. Such critical temperatures are also chosen in order to minimize the effects of hidden criticality in the case of weak coupling between the two bands. For the other parameters, we fixed $\lambda_{11}=2.415, \lambda_{22}=1.211, \lambda_{12}=0.001$, and $\Gamma_{1}=\Gamma_{2}=5.0$, while $N(0)$ is fixed by chosen $\kappa_{1}=10$ [14]. In our simulation, we change the partial densities of states $n_{1}$ and $n_{2}$. The main effect of changing these two parameters is the relative variation of the critical temperatures of band activity, i.e., $T_{c 1}$ and $T_{c 2}$. Hence, for $T_{c 1}$ and for $T_{c 2}<T<T_{c}$, the superconductivity in the system survives only due to the two bands, whereas for $T<T_{c 1}$ and any $T_{c 2}$, both bands are active. For temperature such that $T_{c B}<T<T_{c A}$, one of the bands (band $\mathrm{A}$ ) is active while the other (band $\mathrm{B}$ ) is passive. The vortex configurations corresponding to the first band and the second band having a phase winding number $L_{1}$ and $L_{2}$, respectively, are labeled as $\left(L_{1}, L_{2}\right)$. For a given $n_{1}$, the vortex state was obtained after initializing the system with a particular $\left(L_{1}, L_{2}\right)$ configuration at a given value of $H$. The vortex states with $L_{1} \neq L_{2}$, called fractional states, can occur in the range of $n_{1}$ and $n_{2}$ values for which both bands are active.

Figures 1 and 2 show a detailed analysis of how the magnetization curves and vortex states change with magnetic field. The calculation were done for $n_{1}=1-n_{2}=0.334$, for which $T_{c 1}=T_{c 2}=1.0$ and $\gamma=1.024 \times 10^{-3}$. The $M(H)$ magnetization curves are shown in Fig. 1, when $\nu_{1} / \nu_{2}=0.675$

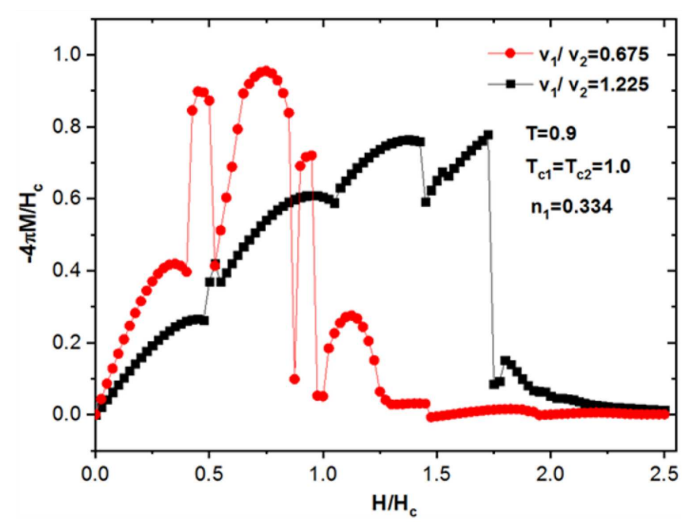

Fig. 1. Magnetization $M(H)$ at $T=0.9$ and $n_{1}=$ $1-n_{2}=0.334$ for the ratio of the Fermi velocities $\nu_{1} / \nu_{2}=0.675$ and $\nu_{1} / \nu_{2}=1.225$, respectively. (a)

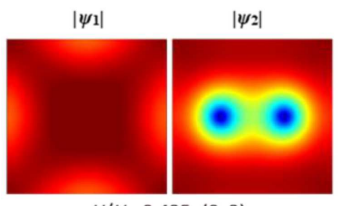

$H / H_{c}=0.425,(0,2)$

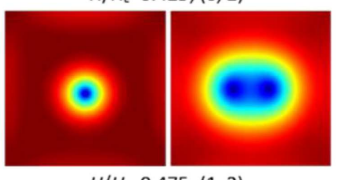

$H / H_{c}=0.475,(1,2)$

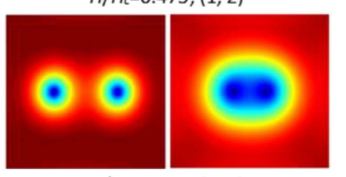

$H / H_{c}=0.500,(2,2)$

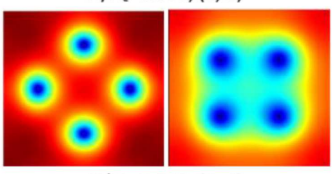

$H / H_{c}=0.650,(4,4)$

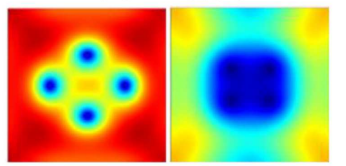

$H / H_{c}=0.900,(4,4)$

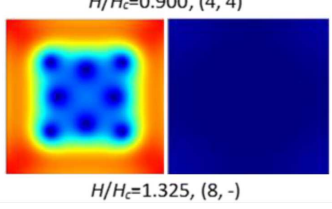

$H / H_{c}=1.325,(8,-)$

Fig. 2. (a) Vortex configurations corresponding to selected states for $\nu_{1} / \nu_{2}=0.675$ and $n_{1}=0.334$. (b) Vortex configurations corresponding to selected states for $\nu_{1} / \nu_{2}=1.225$ and $n_{1}=0.334$.

and $\nu_{1} / \nu_{2}=1.225$, respectively. Figure 1 presents a series of steps corresponding to the entry of vortices, and forming the vortex configurations which are actually shown in Fig. 2a and b, respectively. When $\nu_{1} / \nu_{2}=0.675$, we obtain $\kappa_{1}>\kappa_{2}\left(\kappa_{1}=10.0\right.$ and $\left.\kappa_{2}=3.227\right)$. The vortex entry occurs at first in the second band condensate at $H=0.425 H_{c}$ (see Fig. 2a). Fractional vortices were also found, e.g., $(0,2)$ state at $0.425 H_{c} \leq H<0.475 H_{c}$, and $(1,2)$ state at $0.475 H_{c} \leq \bar{H}<0.500 H_{c}$. When $0.500 H_{c} \leq H<1.325 H_{c}$, no fractional vortex state exists. For the found vortexes, their structures were composite vortex states. This concerns the $(2,2)$ state at $H=0.500 H_{c}$ and the $(4,4)$ state at $H=0.650 H_{c}$ (see Fig. 2a). When $\nu_{1} / \nu_{2}=1.225$, we obtain $\kappa_{1}<\kappa_{2}\left(\kappa_{1}=10.0\right.$ and $\left.\kappa_{2}=10.627\right)$. We can also observe the $(8,12)$ fractional vortex states at $1.525 H_{c} \leq H<1.700 H_{c}$ (see Fig. 2b).

Figures 3 and 4 show the magnetization curves and vortex states change with magnetic field for $n_{1}=1-n_{2}=0.343$, for which $T_{c 1}>T_{c 2}\left(T_{c 1}=1.0\right.$ and $\left.T_{c 2}=0.95\right)$, and $\gamma=0.997 \times 10^{-3}$. Notice that the relative magnetization decreases for $n_{1}=0.343$ as compared to the case for $n_{1}=0.334$. This 


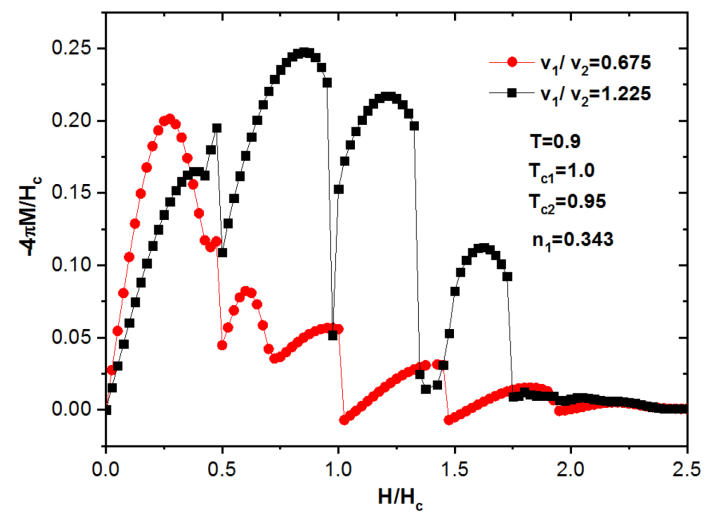

Fig. 3. Magnetization $M(H)$ at $T=0.9$ and $n_{1}=$ $1-n_{2}=0.343$ for the ratio of the Fermi velocities $\nu_{1} / \nu_{2}=0.675$ and $\nu_{1} / \nu_{2}=1.225$, respectively. (a)

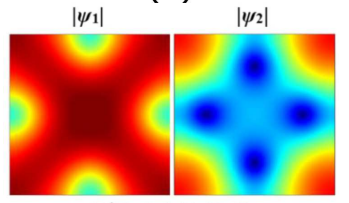

$H / H_{c}=0.475,(0,4)$

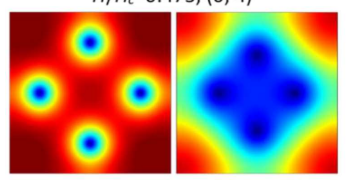

$H / H_{c}=0.500,(4,4)$

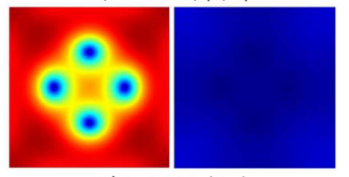

$H / H_{c}=0.800,(4,-)$ (b)

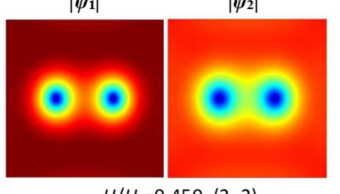

$H / H_{c}=0.450,(2,2)$

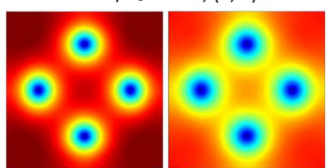

$H / H_{c}=0.550,(4,4)$

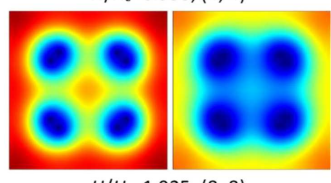

$H / H_{c}=1.025,(8,8)$

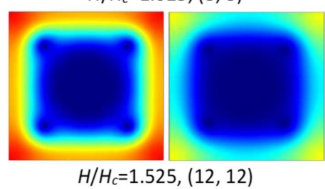

Fig. 4. (a) Vortex configurations corresponding to selected states for $\nu_{1} / \nu_{2}=0.675$ and $n_{1}=0.343$. (b) Vortex configurations corresponding to selected states for $\nu_{1} / \nu_{2}=1.225$ and $n_{1}=0.343$.

may be attributed to the weakening of the strength of the Josephson coupling $\left(\gamma=0.997 \times 10^{-3}\right.$ for $n_{1}=0.343$ while for $n_{1}=0.334$ one has $\left.\gamma=1.024 \times 10^{-3}\right)$. When $\nu_{1} / \nu_{2}=0.675\left(\kappa_{1}=10.0\right.$ and $\left.\kappa_{2}=3.292\right)$, the vortex entry occurs at first in the second band condensate at $H=0.475 H_{c}$. We can only observe the $(0,4)$ fractional vortex state at $0.475 H_{c} \leq H<0.500 H_{c}$. When $H \geq 0.500 H_{c}$, we found the $(4,4)$ composite vortex state only. When $\nu_{1} / \nu_{2}=1.225\left(\kappa_{1}=10\right.$ and $\left.\kappa_{2}=10.843\right)$, no fractional vortex state was found. For the found vortexes, their structures were only the $(2,2),(4,4)$, $(8,8)$ and $(12,12)$ composite vortex states. The vortex configurations corresponding to selected states are shown in Fig. 4.

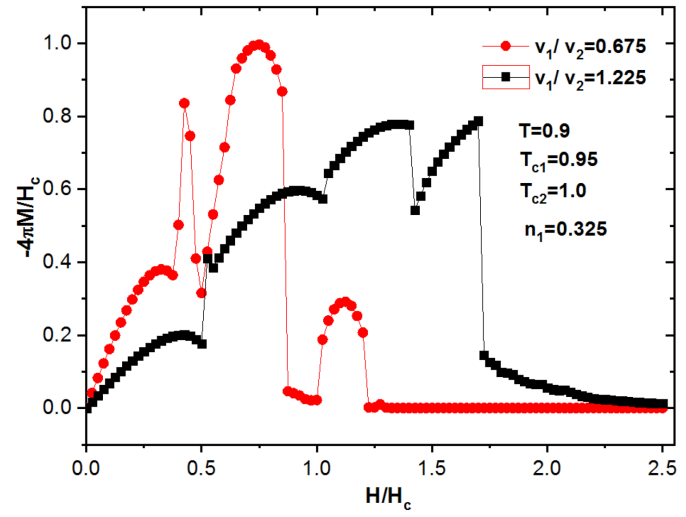

Fig. 5. Magnetization $M(H)$ at $T=0.9$ and $n_{1}=$ $1-n_{2}=0.325$ for the ratio of the Fermi velocities $\nu_{1} / \nu_{2}=0.675$ and $\nu_{1} / \nu_{2}=1.225$, respectively. (a)

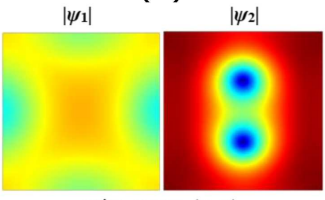

$H / H_{c}=0.425,(0,2)$

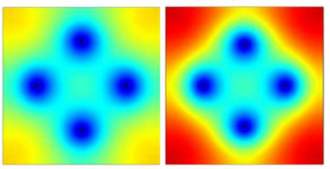

$H / H_{c}=0.550,(4,4)$

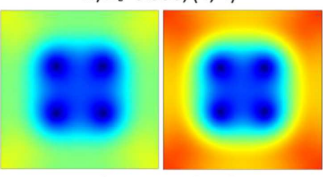

$H / H_{c}=0.825,(4,4)$

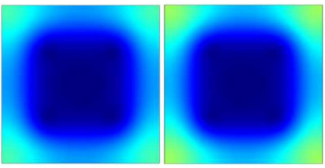

$H / H_{c}=1.125,(8,8)$ (b)

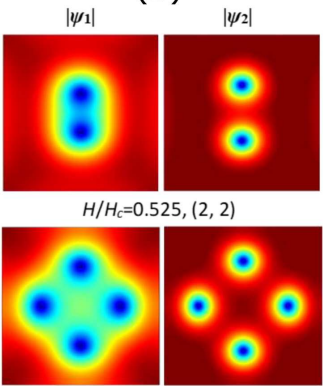

$H / H_{c}=0.625,(4,4)$

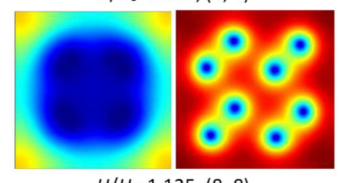

$H / H_{c}=1.125,(8,8)$

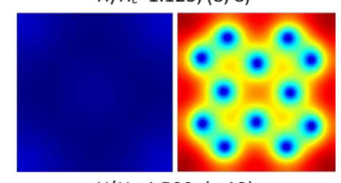

$H / H_{c}=1.500,(-, 12)$
Fig. 6. (a) Vortex configurations corresponding to selected states for $\nu_{1} / \nu_{2}=0.675$ and $n_{1}=0.325$. (b) Vortex configurations corresponding to selected states for $\nu_{1} / \nu_{2}=1.225$ and $n_{1}=0.325$.

Figures 5 and 6 show the magnetization curves and vortex states change with magnetic field for $n_{1}=1-n_{2}=0.325$, for which $T_{c 1}<T_{c 2}$ (i.e., $T_{c 1}=0.95$ and $\left.T_{c 2}=1.0\right)$ and $\gamma=1.052 \times 10^{-3}$. Here we also observed the similar phenomenon for the enhanced coupling effect $\left(\gamma=1.052 \times 10^{-3}\right)$, as compared with $n_{1}=0.334\left(\gamma=1.024 \times 10^{-3}\right)$. When $\nu_{1} / \nu_{2}=0.675\left(\kappa_{1}=10.0\right.$ and $\left.\kappa_{2}=3.162\right)$, the vortex entry first occurs in the second band condensate at $H=0.425 H_{c}$. The only observed fractional vortex state was the $(0,2)$ state at $0.425 H_{c} \leq H<0.600 H_{c}$. When $H \geq 0.600 H_{c}$, no fractional vortex state was found. For the found vortexes, their structures were the $(4,4)$ and $(8,8)$ composite vortex states. When $\nu_{1} / \nu_{2}=1.225$ 


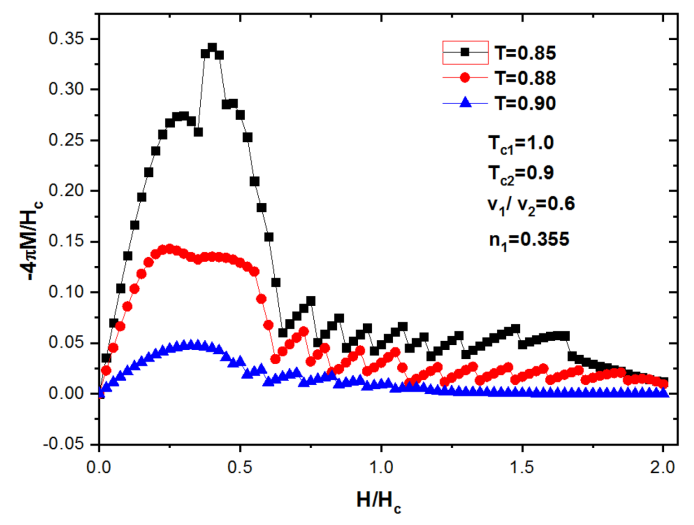

Fig. 7. Magnetization $M(H)$ for $\nu_{1} / \nu_{2}=0.6$ and $n_{1}=1-n_{2}=0.355$ at $T=0.85, T=0.88$, and $T=0.9$, respectively.
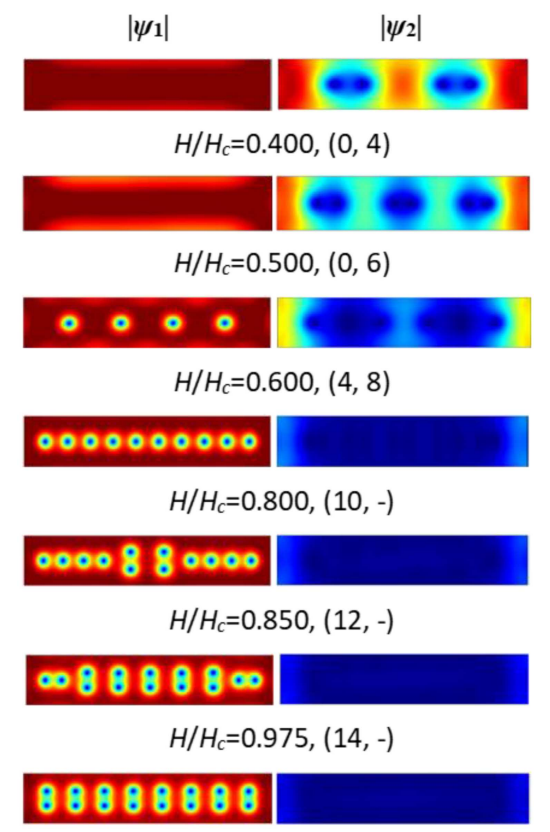

$H / H_{c}=1.075,(16,-)$

888888888

$H / H_{c}=1.225,(20,-)$

Fig. 8. Vortex configurations corresponding to selected states at $T=0.85$ for $\nu_{1} / \nu_{2}=0.6$.

$\left(\kappa_{1}=10\right.$ and $\left.\kappa_{2}=10.413\right)$, the found vortex structures were only the $(2,2),(4,4)$ and $(8,8)$ composite vortex states. The vortex configurations corresponding to the selected states are shown in Fig. 6.

In what follows next, let us consider a mesoscopic superconducting rectangle of the size $L_{x} \times L_{y}=100 \zeta_{1} \times 20 \zeta_{1}$. For the simulation parameters, we take $\lambda_{11}=2.0, \lambda_{22}=1.03, \lambda_{12}=0.005$, $n_{1}=0.355$, and $\Gamma_{1}=\Gamma_{2}=5.0$, while $N(0)$ is fixed by the choice of $\kappa_{1}=10.0$. Now, we have therefore $T_{c 1}=1.00, T_{c 2}=0.90$ and $\gamma=0.0068$. We obtain $\kappa_{1}>\kappa_{2}\left(\kappa_{1}=10.0\right.$ and $\left.\kappa_{2}=2.67\right)$ when $\nu_{1} / \nu_{2}=0.6$, while we obtain $\kappa_{1}<\kappa_{2}\left(\kappa_{1}=10.0\right.$ and $\left.\kappa_{2}=10.683\right)$ when $\nu_{1} / \nu_{2}=1.2$.

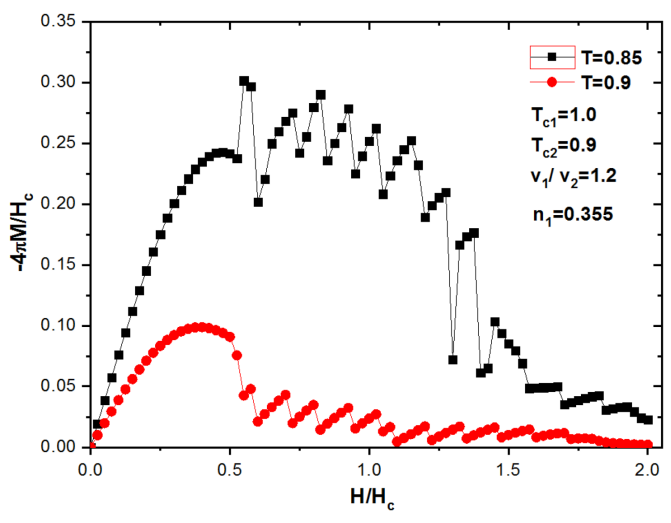

Fig. 9. Magnetization $M(H)$ for $\nu_{1} / \nu_{2}=1.2$ and $n_{1}=1-n_{2}=0.355$ at $T=0.85$ and $T=0.9$, respectively.

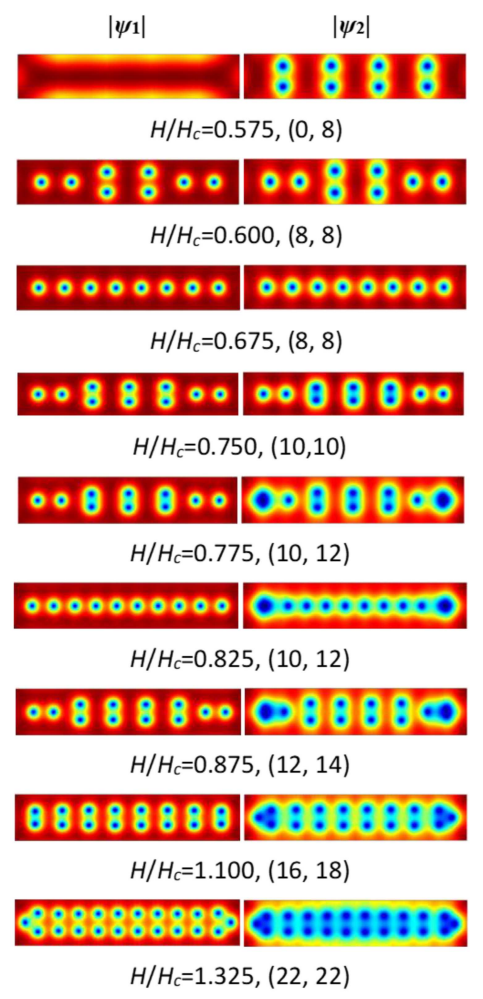

Fig. 10. Vortex configurations corresponding to selected states at $T=0.85$ for $\nu_{1} / \nu_{2}=1.2$.

In Fig. 7, the $M(H)$ magnetization curves in units of the thermodynamic critical field $H_{c}$ at $\nu_{1} / \nu_{2}=0.6$ are shown for different values of temperature $T$ varied from 0.85 to $0.90 \mathrm{~K}$. We can observe the relative magnetization decreases with increasing $T$. The vortex configurations corresponding to selected states at $T=0.85$ are shown in Fig. 8. When $\nu_{1} / \nu_{2}=0.60$ and $T=0.85$, the vortex entry occurs at first in the second band condensate at $H=0.4 H_{c}$. We can observe the $(0,4),(0,6)$ and $(4,8)$ fractional vortex states at $0.4 H_{c} \leq H<0.8 H_{c}$. Importantly, the superconductivity of the second band is suppressed at a lower magnetic field, while the first band still maintains 
superconductivity at the higher magnetic field. When $T=T_{c 2}=0.9$, the second band is very close to the normal state. In fact, the superconductivity still survives in the first band due to the $T<T_{c 1}=1.0$. We can state therefore that the magnetization properties in the system are similar to those of a single band superconductor.

In comparison, the $M(H)$ magnetization curves at $\nu_{1} / \nu_{2}=1.2$ for different $T$ values are shown in Figs. 9 and 10. When $\nu_{1} / \nu_{2}=1.2$ and $T=0.85$, the vortex entry occurs at first in the second band condensate at $H=0.575 H_{c}$. The $(0,8)$ fractional vortex states can be observed at $0.575 H_{c} \leq H<0.6 H_{c}$. In turn, the $(10,12)$, $(12,14)$ and $(16,18)$ fractional vortex states can be observed at $0.775 H_{c} \leqslant H<1.325 H_{c}$.

\section{Conclusions}

Using the time-dependent two-band GinzburgLandau model, we studied the distribution of fractional vortex configurations in the mesoscopic superconductors for different regions of the parameter space. The obtained results revealed that the fractional state could be stable in mesoscopic superconductors with the proper microscopic parameters. The optimized parameter shapes and sizes of the mesoscopic superconductors could maximize the range of the applied magnetic field in which the fractional states are stable. Our results could help to understand and detect the vortex states in mesoscopic multiband superconductors. In addition, it is proved that FEM is a good tool for the study of mesoscopic superconductors. Due to its flexibility and high computing efficiency, it is expected to be extended to the research of superconducting devices with complex geometry.

\section{Acknowledgments}

This work was sponsored by the National Natural Science Foundation of China (61875119) Shanghai Rising-Star Program (Grant No. 19QA1404000) and the Natural Science Foundation of Shanghai (No. 17ZR1411400).

\section{References}

[1] J. Nagamatsu, N. Nakagawa, T. Muranaka, Y. Zenitani, J. Akimitsu, Nature (London) 410, 63 (2001).

[2] P.C. Canfield, G.W. Crabtree, Phys. Today 56, 34 (2003).

[3] K. Ishida, Y. Nakai, H. Hosono, J. Phys. Soc. Jpn. 78, 062001 (2009).
[4] J. Paglione, R.L. Greene, Nature Phys. 6 , 645 (2010).

[5] Y. Lubashevsky, E. Lahoud, K. Chashka, D. Podolsky, A. Kanigel, Nature Phys. 8, 309 (2012).

[6] X.X. Xi, Rep. Prog. Phys. 71, 116501 (2008).

[7] M. Zehetmayer, Supercond. Sci. Technol. 26, 043001 (2013).

[8] V. Stanev, Z. Tešanović, Phys. Rev. B 81 134522 (2010).

[9] E. Babaev, M. Speight, Phys. Rev. B 72, 180502 (2005).

[10] G. Blumberg, A. Mialitsin, B.S. Dennis, M.V. Klein, N.D. Zhigadlo, J. Karpinski, Phys. Rev. Lett. 99, 227002 (2007).

[11] S. Lin, C. Reichhardt, Phys. Rev. B 87, 100508(R) (2013).

[12] L.F. Chibotaru, V.H. Dao, Phys. Rev. B 81, 020502(R) (2010).

[13] S.Z. Lin, L.N. Bulaevskii, Phys. Rev. Lett. 110, 087003 (2013).

[14] R.M. da Silva, M.V. Milošević, D. Domínguez, F.M. Peeters, J. Albino Aguiar, Appl. Phys. Lett. 105, 232601 (2014).

[15] E. Smorgrav, J. Smiseth, E. Babaev, A. Sudbo, Phys. Rev. Lett. 94, 096401 (2005).

[16] J.C. Piña, C.C. de Souza Silva, M.V. Milošević, Phys. Rev. B 86, 024512 (2012).

[17] J. Garaud, J. Carlström, E. Babaev, Phys. Rev. Lett. 107, 197001 (2011).

[18] R.M. da Silva, M.V. Milošević, A.A. Shanenko, F.M. Peeters, J. Albino Aguiar, Sci. Rep. 5, 12695 (2015).

[19] L. Peng, Y. Wang, J. Xu, Y. Zhang, L. Zou, Y. Zhu, C. Cai, Acta Phys. Pol. A 136, 962 (2019).

[20] Y. Wang, L. Peng, J. Lin, Y. Zhang, L. Sang, Acta Phys. Pol. A 137, 1116 (2020).

[21] H.D. Gao, W.W. Sun, J. Comput. Phys. 294, 329 (2015).

[22] L. Peng, Y.J. Wang, J. Xu, Y. Zhang, L. Zhou, Y. Zhu, C. Cai, Acta Phys. Pol. A 136, 962 (2019). 